Communications in Physics, Vol. 20, No. 3 (2010), pp. 377-384

\title{
LARGE-SCALE SYNTHESIS OF CdTe QUANTUM DOTS IN AQUEOUS PHASE
}

\author{
PHAM SONG TOAN AND NGUYEN QUANG LIEM \\ Institute of Material Science, VAST \\ TRINH DUC THIEN \\ Institute of Material Science, VAST \\ and \\ Hanoi National University of Education
}

\begin{abstract}
In this paper, we present the systematically experimental results on the influences of $p H$ of the reaction medium, molar ratio of the precursors on the synthesis in aqueous phase of CdTe quantum dots (QDs) and CdTe/CdS QDs with core/shell structure. Under optimal synthesis conditions, water-soluble CdTe and CdTe/CdS QDs have been prepared that exhibit very strong photoluminescence peaking in the spectral range between $520 \mathrm{~nm}$ and $650 \mathrm{~nm}$ with narrow full width at half maximum ( $\sim 32 \mathrm{~nm}$ in the short-wavelength emission case); depending on the emission range, most samples however exhibit the high luminescence quantum yields ( $\sim 40 \%)$. Moreover, the synthesis in aqueous phase shows some additional advantages: it is possible to prepare high quality CdTe QDs in large-scale (up to gram/reaction) with low cost, less toxic and short production time.
\end{abstract}

\section{INTRODUCTION}

Semiconductor QDs or nanocrystals (NCs) have been intensely studied due to their unique size-dependent properties and their promising applications as well. One of the most highlight applications of semiconductor QDs is that it can be used as fluorescent probes in biomedical labeling and ion detection [1]. In these applications, the water-soluble QDs are required. Another strategic application of semiconductor QDs that requires a large amount of materials is in solid state lighting industry, in which QDs serve as luminnophore matrials to convert blue light from InGaN-based light emitting diodes (LED) into orange emission and then giving rise eventually white light sources.

Many previous literatures have been proved that the hot injection of the $\mathrm{Cd}$ and the Te precursors into the organic solvents such as trioctylphosphine oxide (TOPO), hexadecylamine (HDA) or 1-octadecene (ODE) [2], etc. can make very high quality CdTe QDs whose luminescence quantum yield (QY) can be up to $85 \%$. However, the CdTe QDs prepared with TOPO or other mentioned organic solvent are usually capped by organic ligands that make them water-insoluble. For many biomedical applications, these semiconductor QDs need to be changed into hydrophilic state by doing ligand exchange. In fact, the ligand exchange process requires rather complicated and time-consuming treatments and in many cases it makes the QY decreased. An alternative way to obtain hydrophilic QDs is to synthesize them directly in water [3-6]. In this synthesis, popular chemical 
agents such as cadmium bromide, 3-mecaptopropionic acid (MPA), tellurium powder and sodium borohydride, etc. were used as precursors. The synthesis of semiconductor QDs in water phase is not only simpler, less toxic and more bio-compatible than the hot-injected organometallic ones but also very cost-effective (about 8 times less than that using expensive organic solvents [7]). Moreover, this method is feasible to manufacture QDs in different quantum structure (e.g type II [8]) and is potential to produce in a large scale, just by scale up the starting precursor amounts.

In the synthesis of semiconductor QDs in water phase, influences of $\mathrm{pH}$ of the reaction medium, molar ratio of the precursors and surface passivation after making coreonly QDs must be studied in detail. In order to improve the luminescence efficiency and the stability of obtained QDs, their surfaces must be passivated by a suitable material [9]. Luminescence of QDs passivated by inorganic shell is usually more robust than that of organically passivated one since inorganic epitaxial growth can eliminate efficiently both the anionic and cationic dangling bonds on the QDs surface.

In this paper, we report results of our study on the influences of $\mathrm{pH}$ of the reaction medium, molar ratio of the precursors on the synthesis of $\mathrm{CdTe}$ and $\mathrm{CdTe} / \mathrm{CdS}$ core/shell QDs in aqueous phase. Various characterization techniques such as transmission electron microscopy (TEM), X-ray diffraction (XRD), absorption and photoluminescence (PL) spectroscopies were used showing the high quality of the obtained $\mathrm{CdTe}$ and $\mathrm{CdTe} / \mathrm{CdS}$ core/shell QDs.

\section{EXPERIMENTAL}

\section{II.1. Synthesis of CdTe Core}

Sodium borohydride $\left(\mathrm{NaBH}_{4}, 99 \%\right)$, tellurium powder $(\mathrm{Te}, 99.8 \%)$, thiourea $\left(\left(\mathrm{NH}_{2}\right)_{2} \mathrm{CS}\right.$, $97 \%$ ) were purchased from Merck, cadmium bromide ( $\left.\mathrm{CdBr}_{2}, 99 \%\right)$, and 3-mercaptopropionic acid (MPA, 99\%) were purchased from Aldrich. All chemicals were used without additional purification.

Firstly, $160 \mathrm{mg}$ of Te powder and $100 \mathrm{mg}$ of $\mathrm{NaBH}_{4}$ were put into a two-neck flask and were degassed for $30 \mathrm{~min}$ and backfilled with nitrogen gas. And then, $2 \mathrm{ml}$ of degased distilled water was added into the flask through a syringe. The reaction mixture was mixed by the ultrasonic generator for 30 minutes at the temperature of $50-60^{\circ} \mathrm{C}$ to get a deep red transparent solution via the following chemical reaction:

$$
2 \mathrm{NaBH}_{4}+\mathrm{Te}+2 \mathrm{H}_{2} \mathrm{O}=\mathrm{NaHTe}+\mathrm{NaBO}_{2}+11 / 2 \mathrm{H}_{2}
$$

For a typical synthesis of CdTe QDs, $12.5 \mathrm{mM}$ of $\mathrm{CdBr}^{2+}$ solution was mixed with $18.75 \mathrm{mM}$ of MPA solution at the ratio of $\mathrm{Cd}: \mathrm{MPA}=1: 1.5(\mathrm{~mol} / \mathrm{mol})$ and the $\mathrm{pH}$ of this solution was adjusted in range of $7-12$ by addition of $1.0 \mathrm{M} \mathrm{NaOH}$ solution. After that, the freshly prepared $0.625 \mathrm{M}$ NaHTe solution was injected quickly by a syringe into the $\mathrm{Cd}$ containing flask at the room temperature in nitrogen gas protection. The reaction mixture changes instantly to golden yellow indicating the commencement of CdTe nucleation. The as-prepared CdTe QDs emitted very weak luminescence peaking around $510 \mathrm{~nm}$. 


\section{II.2. Synthesis of CdTe/CdS Core/shell Structure}

To stabilize the physical characteristics of QDs, excessive amounts of thiourea were added into the solution containing CdTe cores and then the solution was annealed at $120^{\circ} \mathrm{C}$ in few minutes or few hours to reach the expected average size. Herein, thioure was used as the sulfur source due to its decomposition at high temperature and the $\mathrm{S}^{2-}$ ions could easily combine with the excessive $\mathrm{Cd}^{2+}$ ions in the initial solution to form $\mathrm{CdTe} / \mathrm{CdS}$ core/shell structures.

\section{II.3. Characterization}

The absorption and PL spectra were obtained by using Cary 5000 (Varian) UVvis-NIR spectrophotometer and iHR550 (Horiba) spectrometer equipped a CCD detector (Synapse), respectively. In the PL measurement, a 377-nm LED was used as the excitation source. The room-temperature PL QY of the prepared samples was estimated by comparing the total integrated emission of the samples with that of Rhodamine $6 \mathrm{G}$ at the same optical density [10]. The quantum yield of Rhodamine $6 \mathrm{G}$ has already been known $\sim 95 \%$ [11]. The powder XRD patterns were received by using a Siemens D5000 powder X-ray diffractometer equipped with a graphite monochromatized high-intensity $\mathrm{Cu} \mathrm{K} \alpha$ radiation $(\alpha=1.54178 \AA$ ). The TEM images were taken by a TEM system (Tecnai 20ST, Osaka University, Japan).

\section{RESULTS AND DISCUSSION}

\section{III.1. Influences of pH Value at the Cd:Te Ratio of 1:0.2}

The $\mathrm{pH}$ of the reaction environment is an important factor to prepare CdTe QDs in aqueous phase. To investigate effects of the $\mathrm{pH}$ value, we fixed the molar ratio of Cd:MPA:Te at 1:1.5:0.2 for completed reaction. In the solution of MPA and $\mathrm{Cd}^{2+}$ mixture, the Cd-MPA complexes were formed. It is supposed that the Cd-MPA complexes could show the polymerization in low $\mathrm{pH}$ environments. Therefore, these complexes are insoluble in acidic media due to the existence of Cd-thiol in the polymer state. On the other hand, in high $\mathrm{pH}$ environments $(\mathrm{pH} \geq 7)$, the Cd-MPA complexes can exist in the molecular state, and easily to be the $\mathrm{Cd}^{2+}$ sources.

In Figure 1, the absorption spectra of various CdTe QDs samples synthesized at different $\mathrm{pH}$ (from 7 to 12) of the reaction medium are shown. It is realized that the CdTe QDs prepared in neutral media (with $\mathrm{pH} \sim 7-8$ ) show sharper, narrower excitonic peak and suggested to have the better crystal quality. With increasing the $\mathrm{pH}$ value of the reaction medium, the excitonic peak from CdTe QDs becomes unclear and broader. Therefore, we suggest that $\mathrm{Cd}^{2+}$ :MPA could be the most effective $\mathrm{Cd}^{2+}$ source for the formation of CdTe QDs in the reaction environment of $\mathrm{pH} \sim 7-8$.

\section{III.2. Influences of the Molar Ratio between MPA and $\mathrm{Cd}$ at $\mathrm{pH}=7$ of the Reaction Environment}

As mentioned, the Cd-MPA complexes take the important role as the $\mathrm{Cd}^{2+}$ source for the formation of CdTe QDs. The generation of these complexes strongly depends on the molar ratio between MPA and $\mathrm{Cd}^{2+}$. Figure 2 a shows the absorption spectra of CdTe QDs prepared with different molar ratio of $\mathrm{Cd}: \mathrm{MPA}$ at the same $\mathrm{pH}=7$ of the 


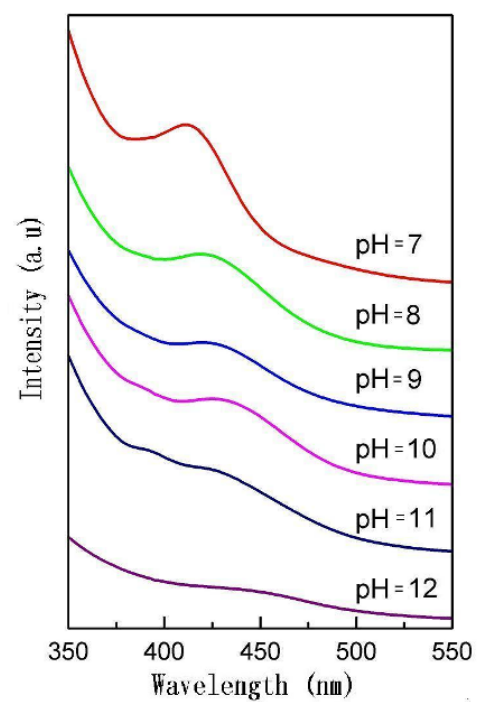

Fig. 1. Absorption spectra of CdTe QDs (Cd:MPA:Te of 1:1.5:0.2) in different $\mathrm{pH}$ values of the reaction environments.
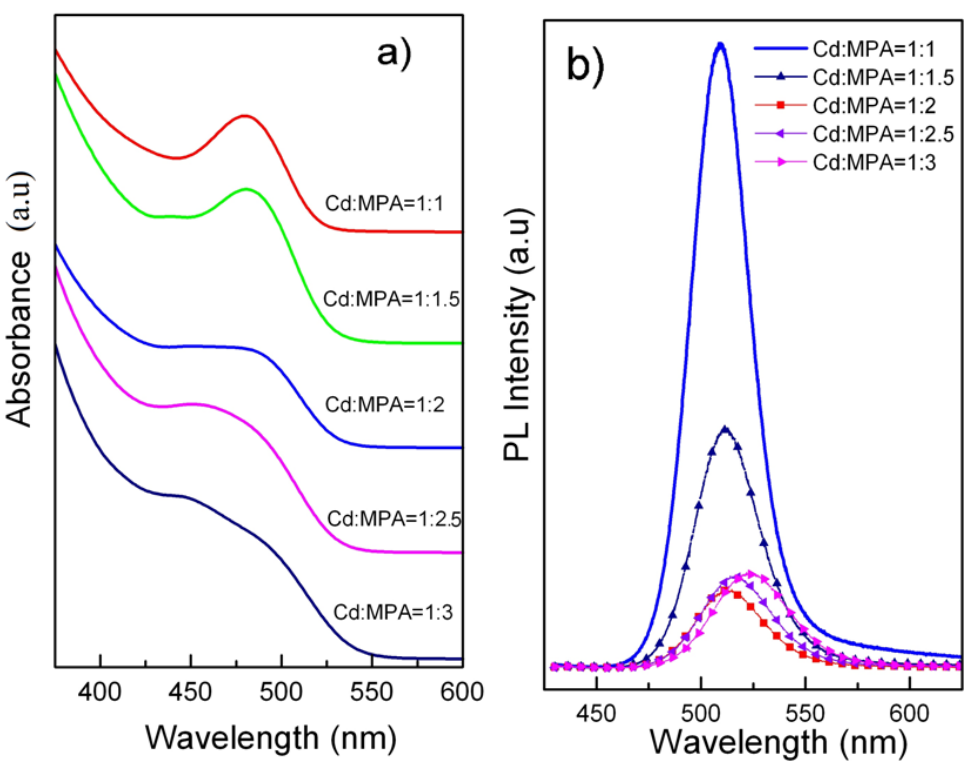

Fig. 2. Absorption and PL spectra of CdTe QDs with different molar ratio of $\mathrm{Cd}$ and MPA (Cd:Te ratio of 1:0.5; $\mathrm{pH}=7$ ).

reaction environment. In addition, the steady PL spectra of these samples with almost the same absorbance were presented in Figure 2b. It is obvious that the samples prepared with Cd:MPA ratio from 1:1 to 1:1.5 exhibit sharper excitonic absorption peaks and more robust $\mathrm{PL}$ signal. These results suggest that at this Cd:MPA ratio range, the generated Cd-MPA 
complexes were the most effective $\mathrm{Cd}^{2+}$ source. To explain such kind of behaviors, we suppose the mechanism of the formation of Cd-thiol complexes as the following equations:

$$
\begin{gathered}
R S H+O H^{-} \rightarrow S R^{-}+H_{2} 0 \\
C d^{2+}+S R^{-} \rightarrow(C d-S R)^{+} \\
(C d-S R)^{+}+S R^{-} \rightarrow C d(S R)_{2}
\end{gathered}
$$

The generated thiolate ions in the eqn.(1) can react with $\mathrm{Cd}^{2+}$ ions to form monothiol complexes following the eqn.(2). In addition, the monothiol complexes can further react with other thiolate ions to create dithiol complexes as described in eqn.(3). As a result, when the Cd:MPA ratio is more than 1:1.5, the amount of the monothiol complexes will be dominant than that of dithiol complexes. On the other hand, when the Cd:MPA ratio is equal to or less than 1:2, the dithiol complexes might be dominant in the Cd-MPA complexes. It is easy to understand that the free $\mathrm{Cd}^{2+}$ ions could be released more easily from monothiol complexes than from dithiol complexes. Therefore, higher MPA ratios limited the release of the free $\mathrm{Cd}^{2+}$ ions for participation in the nucleation and crystal growth of CdTe QDs [12].

\section{III.3. Influence of the Cd-to-Te ratio}

The Cd-to-Te ratio is the main factor which determines the concentration of QDs in the solution. When this ratio is changed, the average size of CdTe QDs is nearly the same. Figure 4 shows the absorption spectra of CdTe QDs samples prepared with different ratio of $\mathrm{Cd}$ :Te and annealed in 5 minutes at $120^{\circ} \mathrm{C}$. The obvious absorption peaks were observed and almost unchanged at $460 \mathrm{~nm}$. Only the size distribution and the concentration of QDs in solution were increased proportionally with increasing reacted Te molecules. When the $\mathrm{Cd}$ :Te ratio is smaller than 1 , the black precipitations attributed to the oxide of tellurium was observed. Therefore, the $\mathrm{Cd}$ :Te should be chosen to be larger than 1 . In addition, the excessive Cd molecules are necessary for further coating CdS. Moreover, the concentrations of the precursors have small effects on the growth rate and optical properties of CdTe QDs and can be negligible [12]. For this reason, it enables the convenient large-scale production of CdTe QDs.

\section{III.4. X-ray diffraction spectrum and TEM images}

Based on the obtained results, high PL QY ( 40\%) CdTe QDs samples could be produced in the neutral media of $\mathrm{pH} \sim 7$ with molar ratio of $\mathrm{Cd}$ :MPA:Te about 1:1.5:0.5. Figure 4 shows the PL spectra of Rhodamin 6G and that of CdTe QDs used to determine the PL QY. With core/shell structure, PL QY of CdTe/CdS QDs increased significantly.

We have prepared CdTe QDs with different sizes for different spectral emissions. Figure 5 shows the flashed-photos of typical CdTe QDs obtained with different annealing times ranging from 30 minutes to 6 hours.

It is obvious that after making the CdS shell to form $\mathrm{CdTe} / \mathrm{CdS}$ core/shell structures the PL QY of QDs increased. Figure 6a shows the XRD patterns of the CdTe/CdS sample annealed in 540 minutes at $120^{\circ} \mathrm{C}$, in which the peaks at diffractive angles of $24.16^{\circ}$, $40.31^{\circ}$ and $46.82^{\circ}$ were observed corresponding to the cubic zinc-blende CdTe and CdS nanocrystals. Thus, in nano-sized cubic CdTe were formed instead of hexagonal one. Note 


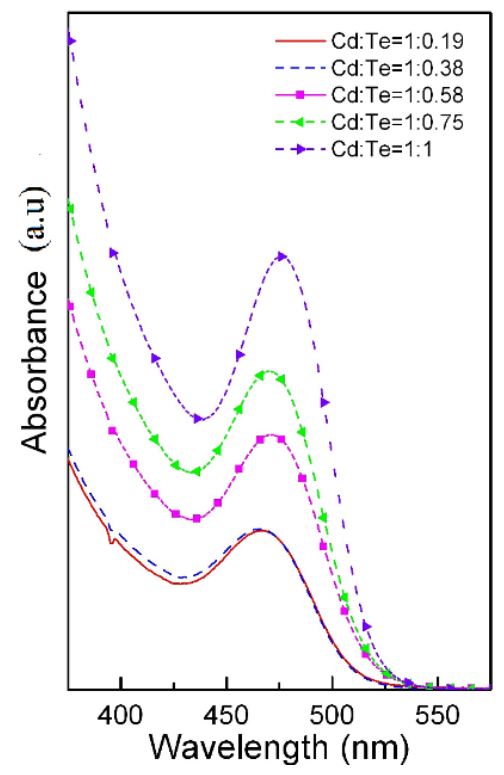

Fig. 3. Absorption spectra of $\mathrm{CdTe}$ QDs $(\mathrm{Cd}: \mathrm{MPA}=1: 1.5 ; \mathrm{pH}=7)$ with different molar ratio of $\mathrm{Cd}$ and $\mathrm{Te}$.

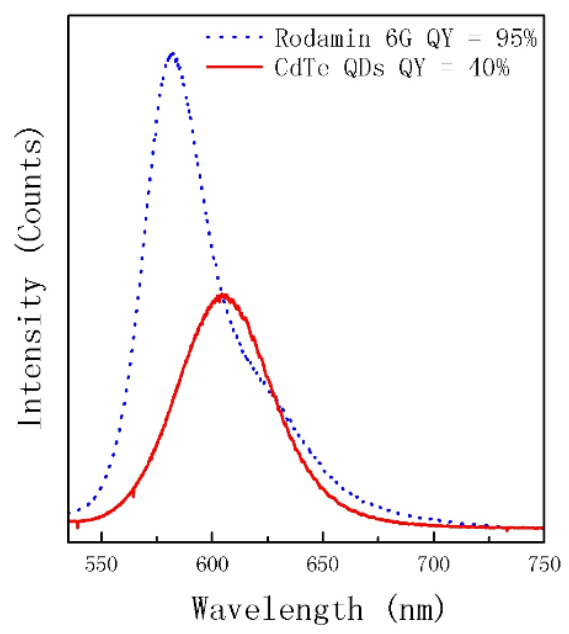

Fig. 4. PL spectra of Rh6G and CdTe QDs used to determine the QY of CdTe QDs; namely $\sim 40 \%$ in this case.

that the XRD pattens may include background caused by ligand or unwanted substance which is hard to remove completely.

In addition, the TEM image of another sample was presented in Figure 6b. From this image, the average size of QDs is observed about 2-3 $\mathrm{nm}$. 


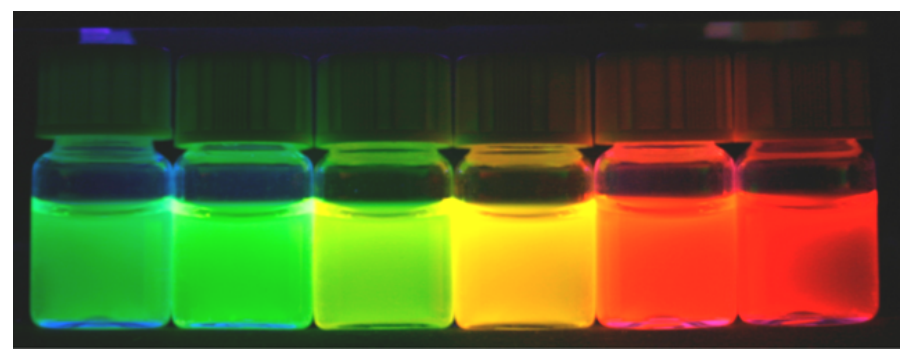

Fig. 5. Typical CdTe QDs samples with different annealing times from from 30 min, $60 \mathrm{~min}$, $90 \mathrm{~min}, 150 \mathrm{~min}, 240 \mathrm{~min}$, and $360 \mathrm{~min}$ from left to right, respectively.

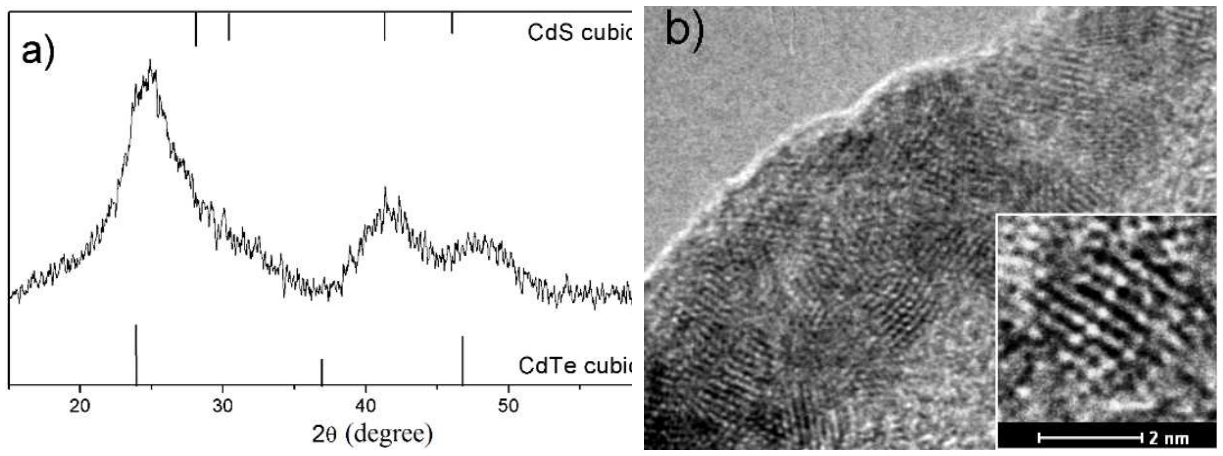

Fig. 6. XRD patterns of CdTe QDs annealed in 540 minutes (a) and TEM image of CdTe QDs annealed in 360 minutes (b)

\section{CONCLUSIONS}

In conclusion, we have studied the influences of the $\mathrm{pH}$ value and molar ratio between the $\mathrm{Cd}$ and the $\mathrm{S}$ precursors on the quality of $\mathrm{CdTe}$ and $\mathrm{CdTe} / \mathrm{CdS}$ QDs. By doing systematically the experiments we have determined the optimal parameters for making high quality CdTe and CdTe/CdS QDs. These QDs synthesized in aqueous phase showed average sizes in the range of $2-3 \mathrm{~nm}$, correspondingly emitted luminescence in the range between $520 \mathrm{~nm}$ and $650 \mathrm{~nm}$ with high QY of $40 \%$.

\section{ACKNOWLEDGEMENTS}

The authors thank L. Q. Phuong for taking the TEM images and UTD Thuy for her help in checking the manuscript. The Basic Research Programme in Natural Science (MOST of Vietnam) and the Materials Science Direction (VAST) are gratefully acknowledged for financial supports.

\section{REFERENCES}

[1] W. Chen, G. A. Joly, S. P. Wang, Luminescence of Semiconductor Nanoparticles. In Encyclopedia of Nanoscience and Nanotechnology, 4 (2004) 689-718.

[2] Andrey L. Rogach (Ed.), Springer Wien New York, (2008). 
[3] Gaponik N, Talapin D V, Rogach A L, Hoppe K, Shevchenko E V, Kornowski A, Eychmuller A and Weller H J, Phys. Chem. B, 106 (2002) 7177.

[4] Zhang H, Wang L, Xiong H, Hu L, Yang B and Li W, Adv. Mater, 15 (2003) 1712.

[5] Zhenyu Gu, Lei Zou, Zheng Fang, Weihong Zhu and Xinhua Zhong, Nanotechnology, 19 (2008) 135604

[6] Yan Liu, Qihui Shen, Dongdong Yu, Weiguang Shi, Jixue Li, Jianguang Zhou, and Xiaoyang Liu, Nanotechnology, 19 (2008) 245601.

[7] Dmitri V. Talapin, PhD thesis, (2002).

[8] Ruosheng Zeng, Tingting Zhang, Jincheng Liu, SongHu, Qiang Wan, Xuanming Liu, Zhiwei Peng, and Bingsuo Zou, Nanotechnology, 20 (2009) 95102

[9] Kortan A R, Hull R, Opila R L, Bawendi M G, Steigerwald M L, Carrol P J and Brus L E, J.Am. Chem. Soc., 112 (1990) 1327.

[10] Glenn A. Crosby, James N. Demas, J. Phys. Chem. 75 (1971) 991-1024.

[11] R. F. Kubin and A. N. Fletcher,J. Lumin., 27 (1983) 455-462.

[12] Lei Zou, Zhenyu Gu, Nan Zhang, Yuliang Zhang, Zheng Fang, Weihong Zhu and Xinhua Zhong, J. Mater. Chem.,18 (2008) 2807-2815. 